\title{
Enhancement of bovine oocyte fertilization in vitro with a bovine oviductal specific glycoprotein
}

\author{
N. S. Martus ${ }^{1}$, H. G. Verhage ${ }^{2}$, P. A. Mavrogianis ${ }^{2}$ and \\ J. K. Thibodeaux ${ }^{\text {I* }^{*}}$ \\ ${ }^{I}$ In Vitro Fertilization Laboratory, Hillcrest Medical Center, Tulsa Center for Fertility and Women's Health, \\ Tulsa, OK 74104, USA; and ${ }^{2}$ Department of Obstetrics and Gynecology, University of Illinois, Chicago, \\ IL 60612, USA
}

\begin{abstract}
The purpose of this study was to determine the effect of a partially purified bovine oviductal glycoprotein (bOGP) on fertilization rates of bovine oocytes. The effect of albumin (control protein) or bOGP at $100 \mu \mathrm{g} \mathrm{ml}^{-1}$ during the $16-18 \mathrm{~h}$ fertilization period was evaluated in a standard IVF system using a sperm concentration between 0.5 and $0.125 \times 10^{6}$ spermatozoa $\mathrm{ml}^{-1}$. bOGP maintained a higher $(P<0.05)$ fertilization rate $(62.0 \%$ versus $31.2 \%)$ at $0.125 \times 10^{6}$ spermatozoa $\mathrm{ml}^{-1}$ compared with the albumin control. The enhancement of fertilization by bOGP was blocked by the inclusion of a specific antibody to bOGP, whereas the antibody with albumin had no effect. A $2 \mathrm{~h}$ gamete preincubation step was subsequently included in the IVF procedure $\left(0.125 \times 10^{6}\right.$ spermatozoa ml $\left.{ }^{-1}\right)$ to determine whether the effect of bOGP was mediated through an interaction with the oocyte, the spermatozoon or both. When oocytes were preincubated with bOGP the fertilization rates were higher $(P<0.05$ ) than with the albumin control (oocytes and spermatozoa exposed to albumin), whereas preincubation of spermatozoa with bOGP did not affect fertilization rates. There was no synergistic effect of preincubating oocytes and spermatozoa with bOGP. The increase in fertilization rate achieved by preincubating oocytes with bOGP was blocked with a specific antibody to bOGP. These results suggest that the increase in fertilization rates observed when bOGP is included during the 16-18 $\mathrm{h}$ fertilization period are primarily mediated through the interaction of bOGP with the oocyte since the same facilitatory effect was observed with a $2 \mathrm{~h}$ preincubation of oocytes before IVF. The ability to block these effects with a polyclonal antibody specifically generated against bOGP shows that this biological activity is due to bOGP. In summary, bOGP enhances fertilization in bovine oocytes whether it is included during preincubation or insemination and this appears to be due to a direct effect on the oocyte.
\end{abstract}

\section{Introduction}

The mammalian oviduct is the site of fertilization and the initial stages of embryonic development. A high molecular weight oviductal glycoprotein (OGP) is synthesized and secreted by the secretory cells of the oviduct. The presence of OGP is highest during the periovulatory period and declines thereafter, suggesting a potential role during in vivo fertilization and the initial cleavage stages (for a review see Malette et al., 1995; Verhage et al., 1997).

OGP has been detected in fluid collected from the oviductal lumen of several mammalian species including baboons (Verhage et al., 1989), humans (Verhage et al., 1988; Rapisarda et al., 1993), hamsters (Araki et al., 1987; Robitaille et al., 1988; Malette et al., 1995), mice (Kapur and Johnson, 1985), rabbits

*Correspondence.

Received 13 October 1997
(Oliphant et al., 1984), pigs (Buhi et al., 1990), sheep (Gandolfi et al., 1989; Buhi et al., 1991; Murray, 1992) and cows (Boice et al., 1990a; Gerena and Killian, 1990). Furthermore, OGP has been shown to associate with the zona pellucida of oocytes in hamsters (Kan et al., 1990; Abe et al., 1992), pigs (Buhi et al., 1993), sheep (Gandolfi et al., 1989) and baboons (Boice et al., $1990 \mathrm{~b}$ ) in addition to the perivitelline space and blastomere membranes of embryos from hamsters, pigs, sheep and baboons (see review by Malette $e t$ al., 1995; O'Day-Bowman et al., 1995). In cattle, OGP has been shown to associate with the zona pellucida and perivitelline space of oocytes and the plasma membrane of oviductal embryos (Wegner and Killian, 1991; Boice et al., 1992). Oviductal glycoprotein has also been shown to associate with bovine (King and Killian, 1994) and hamster (Boatman and Magnoni, 1995) spermatozoa during incubation in vitro, which may enhance bovine sperm capacitation (King et al., 1994) and maintain bovine sperm motility and viability (Abe et al., 1995). Therefore, several studies have 
reported an association of OGP with both gametes. However, the effect of OGP on the fertilization process is unclear.

In bovine IVF systems sperm concentrations are routinely adjusted to maximize fertilization rates and oocytes are often exposed to high concentrations of spermatozoa. The production of oxygen radicals from high concentrations of spermatozoa (Aitken et al., 1996) may adversely affect embryo development and implantation. In addition, during fertilization within the oviduct, oocytes are only exposed to a few spermatozoa (Thibault, 1973).

The present study was conducted to determine the effect of a partially purified bovine OGP (bOGP) on the fertilization rates of bovine oocytes during IVF. After preliminary studies, sperm concentrations were lowered to provide an experimental system more analogous to physiological conditions in vivo.

\section{Materials and Methods}

\section{In vitro maturation and fertilization}

The procedures for in vitro maturation and IVF were as described by Thibodeaux et al. (1995). Cumulus-oocyte complexes harvested from ovaries collected at an abattoir were matured for $22-24 \mathrm{~h}$ while in transit to the laboratory in a portable incubator (Mini-Tube of America, Madison, WI). The maturation medium was Hepes buffered TCM-199 (Sigma Chemical Co., St Louis, MO) supplemented with 10\% (v/v) fetal bovine serum (Hyclone Laboratories, Logan UT), hormones $\left(0.01 \mathrm{iu} \mathrm{ml}^{-1}\right.$ each of purified bovine FSH and $\mathrm{LH}$; NOBL Laboratories, Sioux Center, IA), and antibiotics

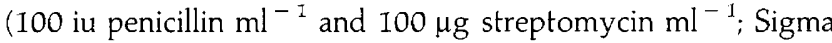
Chemical Co., St Louis, MO).

After the in vitro maturation period, oocytes were pooled and washed twice in Hepes-buffered Tyrode's-lactate (TLHepes) medium (Bavister, 1989). Oocytes were then washed twice in fertilization medium consisting of modified Tyrode'slactate medium (Bavister, 1989) supplemented with $0.25 \mathrm{mmol}$ sodium pyruvate $1^{-1}$ (Sigma Chemical Co., St Louis, MO), antibiotics (100 iu penicillin $\mathrm{ml}^{-1}$ and $100 \mu \mathrm{g}$ streptomycin $\mathrm{ml}^{-1}$ ), and $6 \mathrm{mg}$ fatty acid free BSA $\mathrm{ml}^{-1}$ (Sigma Chemical Co., St Louis, MO; Fert-Talp). Oocytes were transferred into $50 \mu \mathrm{l}$ drops (25-30 oocytes per drop) of fertilization medium in Petri dishes (Falcon; Scientific Products, McGraw Park, IL) that were overlaid with light mineral oil (Sigma Chemical Co. St Louis, MO) previously washed with sterile saline.

Semen from two Holstein bulls was used in the experiments (Eastern Breeders, Ithaca, NY and Select Sires, Plain City, OH). For each group of oocytes, one straw of frozen-thawed semen was layered on a 90 and $45 \%$ discontinuous Percoll gradient and centrifuged at $700 \mathrm{~g}$ for $30 \mathrm{~min}$ as described by Thibodeaux et al. (1995). The sperm pellet was washed twice by centrifugation ( $200 \mathrm{~g}$ for $6 \mathrm{~min}$ ) with fertilization medium and subsequently resuspended in fertilization medium to obtain a stock sperm solution containing $1.0 \times 10^{6}$ spermatozoa ml ${ }^{-1}$. All sperm dilutions were made from the stock solution. A $50 \mu \mathrm{l}$ aliquot of the stock sperm cell suspension was added to the $50 \mu \mathrm{l}$ droplet containing the oocytes.

After $16-18 \mathrm{~h}$ of sperm-oocyte co-incubation at $39^{\circ} \mathrm{C}$ and $5 \% \mathrm{CO}_{2}$ in humidified air, zygotes were transferred through two washes of TL-Hepes to remove spermatozoa. Zygotes were subsequently transferred into Falcon $15 \mathrm{ml}$ conical tubes (Scientific Products, McGraw Park, IL) and the cumulus cells removed by vortexing at high speed for $1 \mathrm{~min}$. After vortexing, the remaining cumulus cells were denuded by manual pipetting and zygotes were mounted on glass slides with coverslips. Slides were placed in fixative (ethanol:acetic acid, 3:1) for at least $24 \mathrm{~h}$ and then stained with $1 \%$ orcein stain (Sigma Chemical Co., St Louis, MO). Slides were evaluated on an inverted microscope $(\times 40)$ to assess normal fertilization (two pronuclei).

\section{Protein preparation}

Bovine oviducts from various stages of the oestrous cycle (Boice et al., 1990a) were collected at an abattoir and placed in sterile saline on ice for transport to the laboratory. Excess connective tissue was removed and the oviducts were then minced and placed in an explant culture system for $24 \mathrm{~h}$ (Verhage et al., 1988). The procedures used for partial purification of bOGP have been described in detail (Boice et al, 1992; Schmidt et al., 1997). Briefly, the proteins present in the culture medium were separated on a Sephacryl S-200 column (Pharmacia LBK, Piscataway, NJ). The proteins eluting in the void volume were dialysed against Millipore water (Milford, MA) using 50000 molecular weight cut-off tubing (Spectra/ Por, Spectrum, Houston, TX) and were then sterile filtered. Protein concentrations were measured using the method of Lowry et al. (1951). Partially purified bOGP was aliquoted, lyophilized and stored at $-20^{\circ} \mathrm{C}$.

\section{Antibody production}

The procedures for the production of a polyclonal antibody to bOGP were as described by Boice et al. (1990a). Oviductal tissue culture media proteins present in the void volume fraction of the S-200 column were separated by onedimensional (1-D) SDS-PAGE on $5 \%$ acrylamide gels and were immediately transferred to nitrocellulose membranes (BA 85, Schleicher and Schuell, Keene, NH) at $200 \mathrm{~mA}$ constant current for 14-18 h using a Hoefer Trans-Blot system (Hoefer Scientific, San Francisco, CA) according to the procedure of Towbin et al. (1979). The nitrocellulose membranes were stained with Ponceau S (Sigma Chemical Co., St Louis, MO) to visualize proteins and the upper band of a doublet at $97 \mathrm{kDa}$ was cut out and dissolved in dimethyl sulfoxide (DMSO). Approximately $100 \mu \mathrm{g}$ of protein was dissolved in $500 \mu \mathrm{l}$ DMSO and an equal volume of Freund's complete adjuvant. A male rabbit was bled from the ear vein and then injected with the protein emulsion at multiple intradermal sites along the back. Booster injections were given at 2, 4, 6, 7 and 8 weeks after the initial injection. Booster injections were approximately $100 \mu \mathrm{g}$ of protein dissolved in DMSO emulsified in Freund's incomplete adjuvant. The antibody used in this study was from blood drawn 10 and 12 weeks after the initial injection. Blood from these collections was pooled and the IgG fraction was purified by ammonium sulphate precipitation followed by ion-exchange chromatography on diethylamino ethyl cellulose (DEAE cellulose; Sigma Chemical Co., St Louis, MO). The IgG 
fraction was further purified by affinity chromatography on bovine serum-agarose (Sigma Chemical Co., St Louis, MO) to remove any antibodies to serum proteins. The isolated $\lg G$ fraction (anti-bOGP IgG) was dialysed against water and sterile filtered. Total protein concentration was measured by the procedure of Lowry et al. (1951), and aliquots were then lyophilized and stored at $-20^{\circ} \mathrm{C}$.

\section{Western blot analysis}

Proteins (approximately $25 \mu \mathrm{g}$ ) present in the void volume fraction of the S-200 column were separated by 1-D SDS PAGE on $7.5 \%$ acrylamide gels and were immediately transferred to nitrocellulose membranes as described previously. Anti-bOGP IgG was used as the primary antibody to detect bOGP in the void volume sample of bovine oviductal tissue culture media. Peroxidase conjugated goat anti-rabbit IgG (BioRad, Hercules, CA) was used as the second antibody and binding was detected using the BioRad peroxidase anti-peroxidase kits according to the manufacturer's instructions.

\section{Experiment 1}

Experiment 1 examined the effects on fertilization rates of inclusion of bOGP in the in vitro insemination period. Preliminary experiments in this study indicated that $100 \mu \mathrm{g}$ bOGP $\mathrm{ml}^{-1}$ was optimal for effects on fertilization and this concentration was used in all experiments. The effects of protein supplement (albumin or bOGP) and final sperm concentration $\left(0.5,0.25\right.$ or $0.125 \times 10^{6}$ spermatozoa $\left.\mathrm{ml}^{-1}\right)$ on fertilization rates were examined by a $2 \times 3$ factorial design. The numbers of oocytes allocated to individual treatment groups were as follows: $0.5 \times 10^{6}$ spermatozoa $\mathrm{ml}^{-1}, 112$ and 113 oocytes for albumin and bOGP, respectively; $0.25 \times 10^{6}$ spermatozoa $\mathrm{ml}^{-1}, 116$ and 126 oocytes for albumin and bOGP, respectively; and $0.125 \times 10^{6}$ spermatozoa $\mathrm{ml}^{-1}, 125$ and 124 oocytes for albumin and bOGP, respectively. A final sperm concentration of $0.5 \times 10^{6}$ spermatozoa $\mathrm{ml}^{-1}$ is used for routine IVF procedures in this laboratory (Thibodeaux et al., 1995). Fertilization dishes were allowed to equilibrate for $\mathrm{Ih}$ at $39^{\circ} \mathrm{C}$ and $5 \% \mathrm{CO}_{2}$ before introduction of oocytes and spermatozoa. Oocytes were placed in the fertilization drops containing protein treatments and the $50 \mu \mathrm{l}$ aliquot of sperm suspension was then added. There was no preincubation of oocytes or spermatozoa with the protein before initiating the experiment.

\section{Experiment 2}

Experiment 2 was conducted to determine whether the enhanced fertilization rates observed in Expt 1 were due to the biological activity of bOGP. Oocytes were randomly assigned to one of the following treatment groups: $100 \mu \mathrm{g}$ albumin $\mathrm{ml}^{-1}$ (170 oocytes), $100 \mu \mathrm{g}$ bOGP $\mathrm{ml}^{-1}$ (187 oocytes), $400 \mu \mathrm{g}$ anti-bOGP IgG ml ${ }^{-1}$ (209 oocytes) or $100 \mu \mathrm{g}$ bOGP and $400 \mu \mathrm{g}$ anti-bOGP IgG ml ${ }^{-1}$ (186 oocytes). Preliminary experiments in this study indicated that $400 \mu \mathrm{g}$ anti-bOGP IgG $\mathrm{ml}^{-1}$ was required to inhibit the effects of $100 \mu \mathrm{g}$ bOGP
Table 1. Experimental design for supplementation of media with bovine oviductal glycoprotein or albumin at different phases in bovine IVF

\begin{tabular}{|c|c|c|c|}
\hline \multicolumn{2}{|c|}{ During $2 \mathrm{~h}$ preincubation } & \multirow{2}{*}{$\begin{array}{c}\text { During } 16-18 \mathrm{~h} \\
\text { insemination }\end{array}$} & \multirow{2}{*}{$\begin{array}{c}\text { Number of } \\
\text { oocytes }\end{array}$} \\
\hline Oocytes & Spermatozoa & & \\
\hline Albumin & Albumin & Albumin & 150 \\
\hline bOGP & Albumin & Albumin & 134 \\
\hline Albumin & bOGP & Albumin & 106 \\
\hline Albumin & Albumin & bOGP & 158 \\
\hline bOGP & bOGP & Albumin & 176 \\
\hline bOGP & Albumin & bOGP & III \\
\hline
\end{tabular}

bOGP: bovine oviductal glycoprotein.

$\mathrm{ml}^{-1}$. Based on the results of Expt 1, the final sperm concentration used was $0.125 \times 10^{\circ}$ spermatozoa $\mathrm{ml}^{-1}$ and all treatment groups were preincubated for $1 \mathrm{~h}$ in the fertilization drops before introducing oocytes and spermatozoa.

\section{Experiment 3}

Experiment 3 examined whether the enhanced fertilization rates observed in Expts 1 and 2 were due to an effect on the oocyte, spermatozoon or both during the insemination process. Oocytes and spermatozoa were exposed to albumin or bOGP during different phases of the IVF process. After the maturation period, oocytes were washed in fertilization medium and randomly assigned to various preincubation treatment groups (Table 1). After the $2 \mathrm{~h}$ preincubation period $\left(39^{\circ} \mathrm{C}\right.$ and $5 \%$ $\mathrm{CO}_{2}$ ), oocytes and spermatozoa were washed in the designated albumin or bOGP treatments to be applied during the 16-18 $\mathrm{h}$ insemination period $\left(39^{\circ} \mathrm{C}\right.$ and $\left.5 \% \mathrm{CO}_{2}\right)$. The insemination medium was supplemented with the albumin and bOGP treatments at a concentration of $100 \mu \mathrm{g} \mathrm{ml}^{-1}$ and the final sperm concentration was $0.125 \times 10^{6}$ spermatozoa ml ${ }^{-1}$.

\section{Experiment 4}

Experiment 4 was conducted to determine whether the enhanced fertilization rates observed after exposure of oocytes to bOGP in Expt 3 were due to the biological activity of bOGP. Oocytes were randomly assigned to one of the following treatment groups: $100 \mu \mathrm{g}$ albumin $\mathrm{ml}^{-1}$ (100 oocytes), $100 \mu \mathrm{g}$ bOGP ml ${ }^{-1}$ ( 90 oocytes) or $100 \mu \mathrm{g}$ bOGP and $400 \mu \mathrm{g}$ anti-bOGP $\mathrm{IgG} \mathrm{ml}{ }^{-1}$ (90 oocytes). Oocytes were preincubated for $2 \mathrm{~h}\left(39^{\circ} \mathrm{C}\right.$ and $\left.5 \% \mathrm{CO}_{2}\right)$, washed in fertilization medium and placed in fertilization drops (containing albumin only). The final sperm concentration was $0.1 \times 10^{6}$ spermatozoa $\mathrm{ml}^{-1}$.

\section{Statistical analysis}

In all experiments each replicate (experimental day consisting of a batch of oocytes) oocytes were randomly assigned across all treatments. All experiments were replicated three times. Data from Expt 1 were analysed by two-way least 


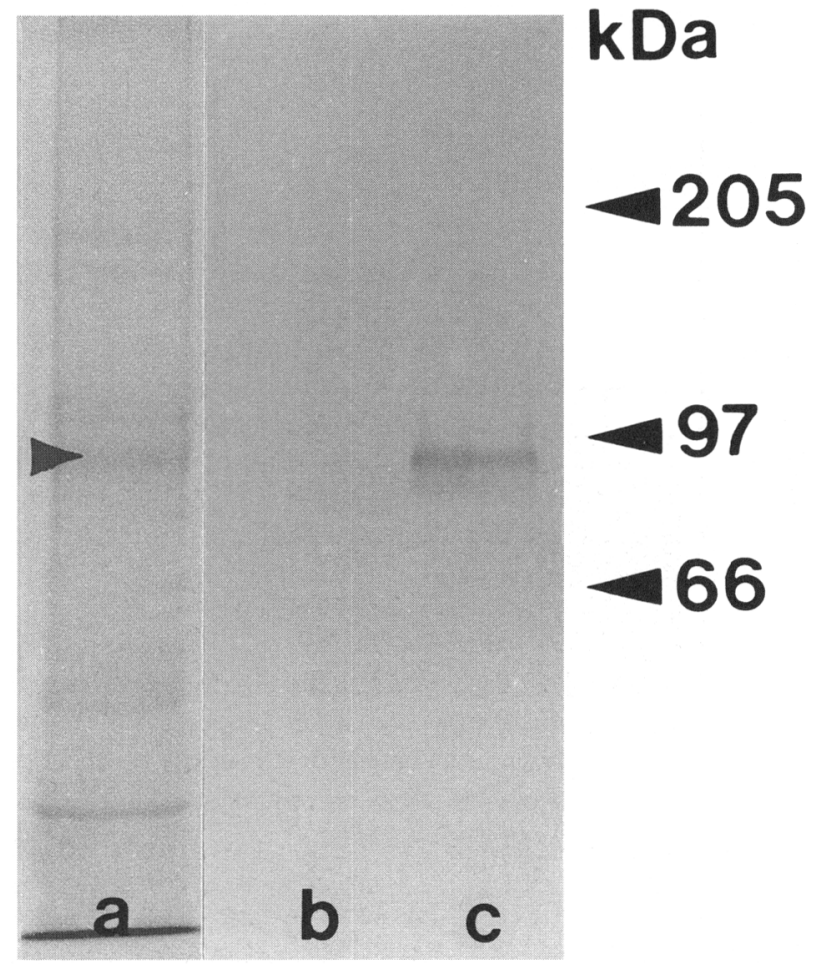

Fig. 1. Serum and partially purified bovine oviductal glycoprotein (bOGP) separated on one-dimensional SDS-PAGE ( $25 \mu \mathrm{g}$ protein per lane). Lane $\mathrm{a}$ is stained with Coomassie blue; lanes $\mathrm{b}$ and $\mathrm{c}$ are immunoblots using the polyclonal antibody anti-bOGP IgG. Lanes a and $c$, partially purified bOGP; lane $b$, bovine serum. Note the major doublet band at approximately $97 \mathrm{kDa}$ in lanes a and $\mathrm{c}$ (arrowhead) and the absence of crossreacting antigens in lane $b$.

squares analysis of variance (ANOVA) with SigmaStat (Jandel Scientific, San Rafael, CA) statistical analysis software. Data from Expts 2, 3 and 4 were analysed by one-way least squares ANOVA. In all experiments, least-square means were compared using Tukey's highest significant difference test, and a probability of $P<0.05$ was considered statistically significant.

\section{Results}

\section{Western blot}

Approximately $25 \mu \mathrm{g}$ of bovine serum protein and partially purified (35-50\% based on SDS-PAGE analysis; Fig. I, lane a) S-200 column void volume fluid from bovine oviductal culture media were analysed by western blotting. Anti-bOGP IgG was used at a concentration of $1.35 \mu \mathrm{g} \mathrm{ml}^{-1}$. A doublet band of crossreacting antigens (Fig. 1) was observed at approximately $97 \mathrm{kDa}$ in the bovine oviduct sample (Fig. 1, lane c), whereas crossreacting antigens were absent in bovine serum (Fig. I, lane b). These results are similar to the observations of other studies using different polyclonal antibodies to bOGP (Boice et al., 1990a; Abe et al., 1993; Gandolfi et al., 1993).

\section{Experiment 1}

The effects of bOGP on fertilization rates were evaluated by reducing the concentration of spermatozoa used during the IVF

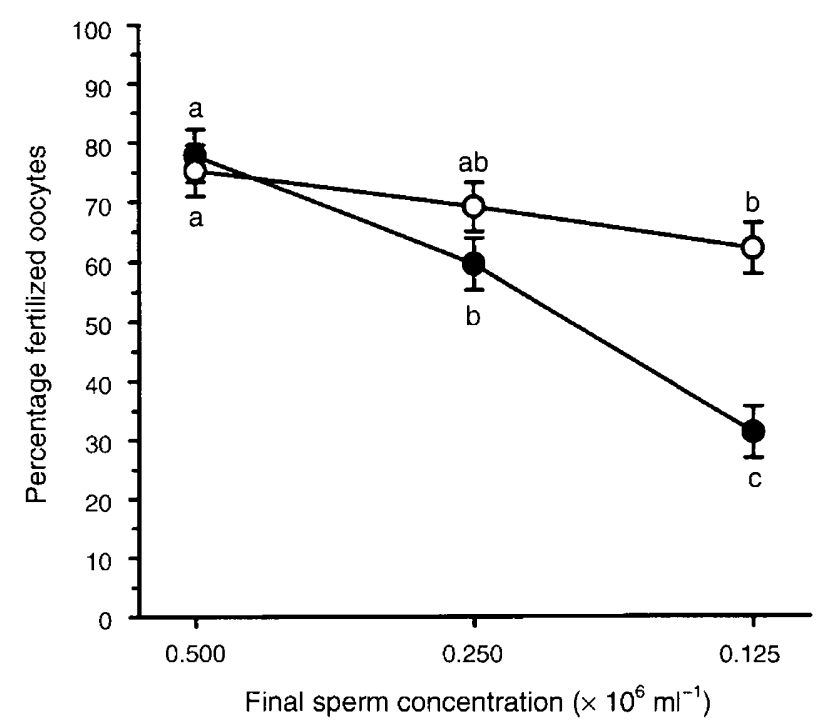

Fig. 2. Effect of bovine oviductal glycoprotein (bOGP) $\left(100 \mu \mathrm{g} \mathrm{ml}^{-1}\right.$ ) and sperm concentration on fertilization rates of bovine oocytes in vitro. (O) bOGP; (O) albumin. Values are least square mean ( \pm SEM) fertilization rates derived from three replicates. Different letters above or below the error bars indicate values are significantly different $(P<0.05)$.

process (Fig. 2). Within the albumin treatment, there was a significant decrease $(P<0.05)$ in fertilization rate with decreasing sperm concentration. Within the bOGP treatment, there was no difference in fertilization rate between the 0.5 and $0.25 \times 10^{6}$ spermatozoa $\mathrm{ml}^{-1}$ and the 0.25 and $0.125 \times 10^{6}$ spermatozoa $\mathrm{ml}^{-1}$ concentrations. However, a decrease $(P<0.05)$ in fertilization rate was observed between the 0.5 and $0.125 \times 10^{6}$ spermatozoa $\mathrm{ml}^{-1}$ concentrations. The albumin treatment had a significantly lower $(P<0.01)$ fertilization rate at $0.125 \times 10^{6}$ spermatozoa $\mathrm{ml}^{-1}$ compared with the bOGP treatment.

\section{Experiment 2}

Experiment 1 demonstrated that incubation of oocytes and spermatozoa with bOGP enhanced fertilization rate at a low sperm concentration $\left(0.125 \times 10^{6}\right.$ spermatozoa $\left.\mathrm{ml}^{-1}\right)$. In Expt 2 the effects of bOGP $\left(100 \mu \mathrm{g} \mathrm{ml}^{-1}\right)$ on fertilization rates were blocked by the inclusion of anti-bOGP IgG (Fig. 3). Oocytes and spermatozoa incubated with bOGP had a significantly higher $(P<0.05)$ fertilization rate compared with all other treatment groups. There were no differences in fertilization rates between the albumin, anti-bOGP IgG, and bOGP and anti-bOGP IgG treatment groups. Therefore, the biological activity of bOGP was completely inhibited by the addition of anti-bOGP IgG during in vitro insemination.

\section{Experiment 3}

Preincubation of oocytes with $100 \mu \mathrm{g}$ bOGP $\mathrm{ml}^{-1}$ for $2 \mathrm{~h}$ before in vitro insemination significantly $(P<0.05)$ increased fertilization rates compared with the control group, whereas 


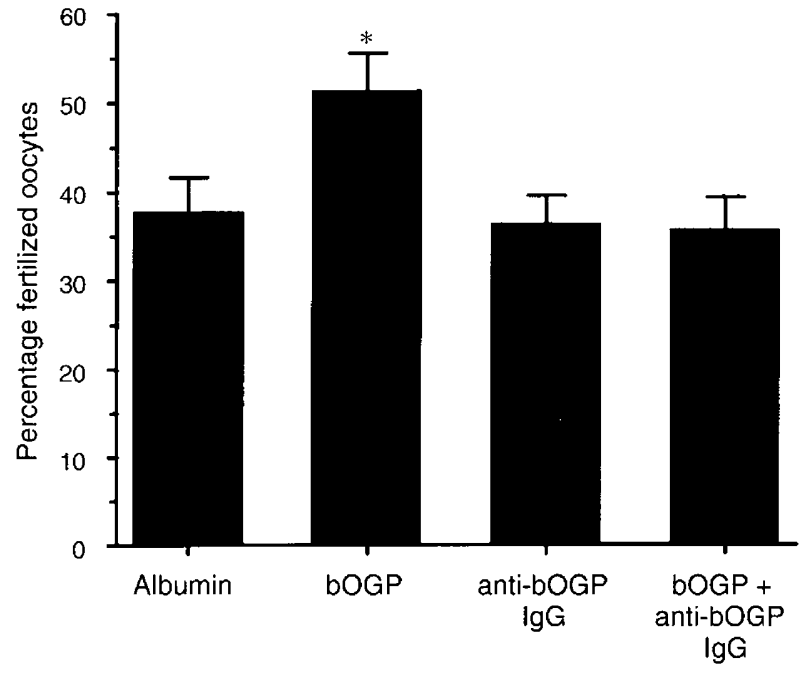

Fig. 3. Effect of bovine oviductal glycoprotein (bOGP) and antibOGP $\operatorname{IgG}$ on fertilization rates of bovine oocytes in vitro using a final sperm concentration of $0.125 \times 10^{6}$ spermatozoa $\mathrm{ml}^{-1}$. The concentration of protein was $100 \mu \mathrm{g} \mathrm{ml}^{-1}$ for the albumin and bOGP treatments and $400 \mu \mathrm{g} \mathrm{ml}^{-1}$ for the anti-bOGP IgG treatment. The bars indicate the least square mean ( \pm SEM) fertilization rates derived from three replicates. ${ }^{*}$ indicates a significant difference $(P<0.05)$ compared with all other treatment groups.

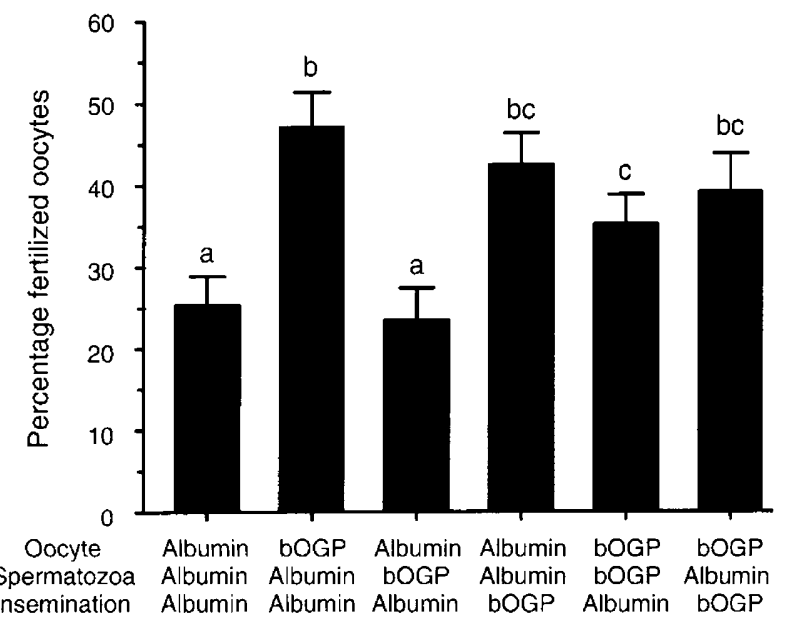

Fig. 4. Effect of bovine oviductal glycoprotein (bOGP) or albumin (both at $100 \mu \mathrm{g} \mathrm{ml}^{-1}$ ) on fertilization rates during different phases of the bovine IVF process using a final sperm concentration of $0.125 \times 10^{6} \mathrm{ml}^{-1}$. Oocytes and spermatozoa were preincubated for $2 \mathrm{~h}$ before insemination with albumin or bOGP, rinsed, and introduced into albumin or bOGP insemination treatments for 16-18 $\mathrm{h}$. The bars indicate the least square mean ( \pm SEM) fertilization rates derived from three replicates. Different letters above the error bars indicate values are significantly different $(P<0.05)$.

preincubation of spermatozoa with bOGP was the same $(P>0.05)$ as observed in the control group (Fig. 4). The presence of bOGP during the $16-18 \mathrm{~h}$ insemination period also significantly $(P<0.05)$ increased fertilization rates compared with the control group, as also observed in Expts 1 and 2 and in the oocyte preincubation group in Expt 3. Preincubating

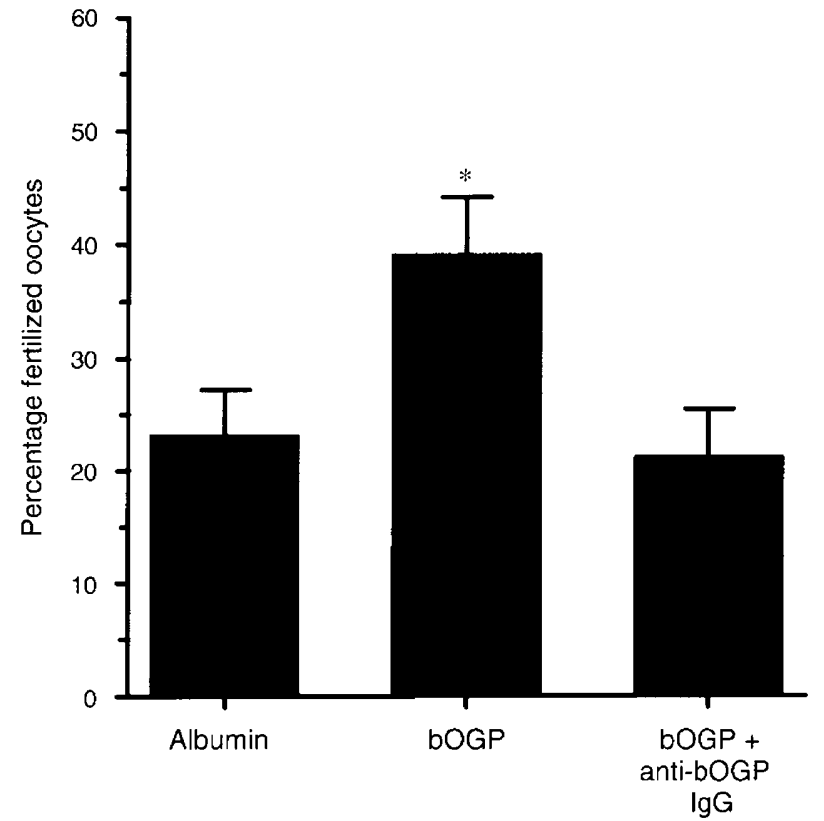

Fig. 5. Effect of bovine oviductal glycoprotein (bOGP) $\left(100 \mu \mathrm{g} \mathrm{ml}^{-1}\right)$ and anti-bOGP $\operatorname{IgG}\left(400 \mu \mathrm{g} \mathrm{ml}{ }^{-1}\right)$ on fertilization rates of bovine oocytes preincubated for $2 \mathrm{~h}$ before IVF using a final sperm concentration of $0.1 \times 10^{6} \mathrm{ml}^{-1}$. Oocytes were washed in albumin and standard IVF was conducted for $16-18 \mathrm{~h}$ in albumin. The bars indicate the least square mean ( \pm SEM) fertilization rates derived from three replicates. ${ }^{*}$ indicates a significant difference $(P<0.05)$ compared with all other treatment groups.

both spermatozoa and oocytes with bOGP also resulted in a significant $(P<0.05)$ increase in fertilization rates, although this was not greater than that observed for preincubation of oocytes. Including bOGP in the oocyte preincubation media and during the 16-18 h insemination period resulted in a significant $(P<0.05)$ increase in fertilization rate, which was the same as that observed when oocytes were preincubated with bOGP.

\section{Experiment 4}

Experiment 3 demonstrated that preincubation of oocytes for $2 \mathrm{~h}$ with bOGP significantly $(P<0.05)$ enhanced fertilization rates compared with the albumin control. In Expt 4 the inclusion of a polyclonal antibody to bOGP (anti-bOGP $\operatorname{lgG}$ ) was used to investigate whether the effect of bOGP on oocytes was due to the biological activity of the glycoprotein. Oocytes preincubated with bOGP had a higher $(P<0.05)$ fertilization rate compared with all other treatment groups (Fig. 5). The effects of bOGP were blocked by the inclusion of anti-bOGP $\operatorname{IgG}$ during the preincubation step.

\section{Discussion}

IVF techniques are well established and the fertilization rates achieved with well defined protocols are 80-90\%. However, oocytes are exposed to very high concentrations of spermatozoa during IVF which may ultimately have a detrimental effect 
on embryonic development and implantation since dying spermatozoa produce oxygen radicals (Aitken, 1995; Aitken et al., 1996). The oviduct probably provides the optimal conditions for early reproductive events, and the ability to mimic this environment may enhance fertilization and early embryonic development and implantation since few spermatozoa are present in the ampulla at the time of fertilization (Hunter, 1988). An important constituent of the oviductal environment is a high molecular weight OGP. This secretory protein is well characterized in several species (for a review see Verhage et al., 1997) including cattle (Boice et al., 1990a, 1992; Gerena and Killian, 1990; Abe et al., 1995).

The results of the present study demonstrated that inclusion of a partially purified bOGP preparation during the $16-18 \mathrm{~h}$ insemination period enhanced fertilization rates compared with albumin controls when sperm concentrations were reduced from 0.5 to $0.125 \times 10^{6}$ spermatozoa $\mathrm{ml}^{-1}$. This effect is probably due to bOGP since preincubating bOGP with antibOGP IgG blocked the effect, whereas anti-bOGP IgG had no effect on fertilization rates. These data support previous reports in other species in which incubation of both gametes with OGP increased sperm binding to ovarian oocytes and sperm penetration rates. Schmidt et al. (1997) reported that the incubation of hamster ovarian oocytes and spermatozoa with hamster OGP in a sperm binding assay resulted in a twofold increase in sperm binding compared with controls. O'Day-Bowman et al. (1996) reported that the co-incubation of human spermatozoa and human zona pellucida with human OGP in a hemizonae assay resulted in a threefold increase in sperm binding compared with controls. In both studies the effect on sperm binding was blocked by specific antibodies generated against human (O'Day-Bowman et al., 1996) and hamster OGP (Schmidt et al., 1997). Boatman and Magnoni (1995) reported that capacitated hamster spermatozoa incubated with ovarian oocytes (not exposed to endogenous OGP) had threefold higher penetration rates in the presence of hamster OGP compared with the controls. Therefore, OGP facilitates sperm binding to the zona pellucida, sperm penetration of the zona pellucida, and fertilization. In addition, including bOGP in IVF protocols may allow sperm concentrations to be significantly reduced while maintaining acceptable fertilization rates, and improve both embryonic development and implantation rates.

Oocytes and spermatozoa were co-incubated with bOGP during the fertilization period in the Expts 1 and 2. Therefore, the improved fertilization rates observed in these experiments could be due to the effect of bOGP on one of the gametes or a synergistic effect on both gametes. Before fertilization in vivo both gametes are exposed to OGP. Several studies have shown that OGP binds to the zona pellucida and perivitelline space of oviductal oocytes (Boice et al., 1990a, 1992; Wegner and Killian, 1991; Kan and Roux, 1995). Binding of OGP to oocytes occurs very rapidly once the oocyte enters the oviduct and the glycoprotein remains associated with the zona pellucida even after vigorous washing (Reuter et al., 1994). OGP has also been reported to associate with bovine (King and Killian, 1994) and hamster (Boatman and Magnoni, 1995) spermatozoa during incubation in vitro. However, in similar studies in vitro, OGP did not associate with human (Reuter et al., 1994) or sheep (Hill and Nancarrow, 1993) spermatozoa. In addition, is it not known whether OGP binds to spermatozoa in any species in vivo.

Preincubation of oocytes with bOGP resulted in a significant increase in fertilization rates which was similar to that observed when bOGP was included in the $16-18 \mathrm{~h}$ fertilization period (oocytes and spermatozoa exposed). However, when spermatozoa were preincubated with bOGP no increase in fertilization rate was observed, nor was there a synergistic effect when both gametes were preincubated with bOGP. The significant increase in fertilization rates observed when oocytes were preincubated with bOGP is likely due to the biological activity of bOGP, since inclusion of anti-bOGP IgG during culture blocked this effect. In addition, Staros and Killian (1997) incubated bovine oocytes for $2.5 \mathrm{~h}$ in bOGP purified from oviductal fluid and observed a significant increase in fertilization rates compared with oocytes incubated in BSA alone. These results suggest that the facilitatory effect of OGP on early reproductive events is primarily mediated through interaction of the protein with the oocyte, possibly through association with the zona pellucida and perivitelline space.

There was no effect on fertilization rates when spermatozoa were preincubated with bOGP. This finding is contradictory to previously published data. King et al. (1994) reported an increase in bovine sperm capacitation during $4 \mathrm{~h}$ incubation in the presence of bOGP compared with controls. The bOGP used in this study was purified from oviductal fluid and used at a concentration of approximately $1 \mathrm{mg} \mathrm{ml}^{-1}$. In addition, spermatozoa capacitated in the presence of bOGP significantly increased fertilization rates compared with spermatozoa capacitated in the absence of bOGP (King et al., 1994). The effect of bOGP was blocked when an antibody generated against bOGP was included in the capacitation media. The discrepancy between the present results and those of King et al. (1994) may be due to different experimental designs. King et al. (1994) used bOGP purified from oviductal fluid and exposed spermatozoa to bOGP during capacitation, whereas in the present study, partially purified bOGP released during culture was used after capacitation. In addition, different bOGP concentrations ( $<100 \mu \mathrm{g} \mathrm{mi} \mathrm{m}^{-1}$ versus approximately $1 \mathrm{mg}$ $\mathrm{ml}^{-1}$ ) and sperm concentrations $\left(0.125 \times 10^{6} \mathrm{ml}^{-1}\right.$ versus $0.5 \times 10^{6} \mathrm{ml}^{-1}$ ) were used. It has also been reported that bOGP maintains sperm motility and viability (Abe et al., 1995). Additional studies are needed in cattle and other species to determine the effect of OGP on sperm physiology. This may not be possible until highly purified samples of endogenous OGP or biologically active recombinant OGP are available.

In conclusion, this study shows that bOGP significantly enhances fertilization rates when sperm concentrations are reduced in current IVF protocols. The observed effect appears to be primarily mediated through the interaction of OGP with the oocyte. Preliminary studies suggest that this effect may also extend to embryonic development (Martus et al., 1997). The role of OGP in fertilization and early embryonic development has important implications for current IVF protocols. Including bOGP in bovine IVF protocols may allow sperm concentrations to be significantly reduced while maintaining acceptable fertilization rates, and improve both embryonic development and implantation rates. 
The research was supported in part by NIH Grant HD20571 (H. G. Verhage) and a grant from the Hillcrest Medical Center Foundation (J. K. Thibodeaux).

\section{References}

Abe H, Ookata K, Abe M and Oikawa T (1992) Immunological characterization of oviductal glycoproteins associated with the zona pellucida of the golden hamster egg Journal of Experimental Zoology 262 209-218

Abe H, Numazawa C, Abe M, Onodera M and Katsumi A (1993) Immunocytochemical localization of oviduct-specific glycoproteins in the bovine oviductal epithelium at follicular and luteal phases Cell Tissue Research 247 41-47

Abe H, Sendai Y, Satoh T and Hoshi H (1995) Bovine oviduct-specific glycoprotein: a potent factor for maintenance of viability and motility of bovine spermatozoa in vitro. Molecular Reproduction and Development 42 226-232

Aitken RJ (1995) Free radicals, lipid peroxidation and sperm function Reproduction, Fertility and Development 7 659-668

Aitken RJ, Buckingham DW, Carreras A and Irvine DS (1996) Superoxide dismutase in human sperm suspensions: relationship with cellular composition, oxidative stress, and sperm function Free Radical Biological Medicine 21 495-504

Araki Y, Kurata S, Oikawa T, Yamahita T, Hiroi M, Naiki M and Sendo F (1987) A monoclonal antibody reacting with the zona pellucida of the oviductal egg but not with that of the ovarian egg of the golden hamster Journal of Reproductive Immunology 11 193-208

Bavister BD (1989) A consistently successful procedure for in vitro fertilization of golden hamster eggs Gamete Research 23 139-158

Boatman DE and Magnoni GE (1995) Identification of a sperm penetration factor in the oviduct of the golden hamster Biology of Reproduction 52 199-207

Boice ML, Geisert RD, Blair RM and Verhage HG (1990a) Identification and characterization of bovine oviductal glycoproteins synthesized at estrus Biology of Reproduction 43 457-465

Boice ML, McCarthy TJ, Mavrogianis PA, Donnelly K, Fazleabas AT and Verhage HG (1990b) Localization of oviductal glycoproteins within the zona pellucida and perivitelline space of ovulated ova and early embryos in baboons (Papio anubis). Biology of Reproduction 43 340-346

Boice ML, Mavrogianis PA, Murphy CN, Prather RS and Day BN (1992) Immuno-cytochemical analysis of the association of bovine oviduct-specific glycoproteins with early embryos Journal of Experimental Zoology 263225 229

Buhi WC, Alvarez IM, Sudhipong V and Dones-Smith MM (1990) Identification and characterization of de novo-synthesized porcine oviductal secretory proteins Biology of Reproduction 43 929-938

Buhi WC, Bazer FW, Alvarez IM and Mirando MA (1991) In vitro synthesis of oviductal proteins associated with estrus and 17-estradiol-treated ovariectomized ewes Endocrinology 128 3086-3095

Buhi WC, O'Brien B, Alvarez IM, Erdos G and Dubois D (1993) Immunogold localization of porcine oviductal secretory proteins within the zona pellucida, perivitelline space, and plasma membrane of oviductal and uterine oocytes and early embryos Biology of Reproduction 48 1274-1283

Gandolfi F, Brevini TAL, Richardson L, Brown CR and Moor RM (1989) Characterization of proteins secreted by sheep oviduct epithelial cells and their function in embryonic development Development 106 303-312

Gandolfi F, Passoni L, Modina S, Brevini TAL, Varga Z and Lauria A (1993) Similarity of an oviduct-specific glycoprotein between species Reproduction, Fertility and Development 5 433-443

Gerena RL and Killian GJ (1990) Electrophoretic characterization of proteins in oviductal fluid of cows during the estrous cycle Journal of Experimental Zoology 256 113-120

Hill JL and Nancarrow CD (1993) Absence of binding of an ovine oestrusassociated oviductal glycoprotein to ram spermatozoa Proceedings of Australian Society of Reproductive Biology 25 73-79

Hunter RHF (1988) Development of the fallopian tubes and their functional anatomy. In The Fallopian Tubes. Their Role in Fertility and Infertility pp 24-42 Ed. RHF Hunter. Springer-Verlag, New York

Kan FWK and Roux E (1995) Elaboration of an oviductin by the oviductal epithelium in relation to embryo development as visualized by immunocytochemistry Microscopic Research Techniques 31 478-487

Kan FWK, Roux E, St-Jacques S and Bleau G (1990) Demonstration by lectingold cytochemistry of transfer of glycoconjugates of oviductal origin to the zona pellucida of oocytes after ovulation in hamsters Anatomical Record 226 $37-47$
Kapur RP and Johnson LV (1985) An oviductal fluid glycoprotein associated with ovulated mouse ova and early embryos Developmental Biology 112 89-93

King RS and Killian GJ (1994) Purification of bovine estrus-associated protein and localization of binding on sperm Biology of Reproduction 51 34-42

King RS, Anderson SH and Killian GJ (1994) Effect of bovine oviductal estrus-associated protein on the ability of sperm to capacitate and fertilize oocytes Journal of Andrology 15 468-478

Lowry OH, Rosebrough NJ, Farr AL and Randall RJ (1951) Protein measurement with the folin phenol reagent Journal of Biological Chemistry 193 265-271

Malette B, Paquette Y, Merlen Y and Bleau G (1995) Oviductins possess chitinase- and mucin-like domains: a lead in the search for the biological function of these oviduct-specific ZP-associating glycoproteins Molecular Reproduction and Development $41384-397$

Martus NS, Verhage HG, Mavrogianis PA and Thibodeaux JK (1997) Enhanced in vitro development of bovine embryos in the presence of a bovine oviductal specific glycoprotein Theriogenology 47 Abstract 334

Murray MK (1992) Biosynthesis and immunocytochemical localization of an estrogen-dependent glycoprotein and associated morphological alterations in the sheep ampulla oviduct Biology of Reproduction $47889-902$

O'Day-Bowman MB, Mavrogianis PA, Fazleabas AT and Verhage HG (1995) A human oviduct-specific glycoprotein: synthesis, secretion, and localization during the menstrual cycle Microscopic Research Techniques 32 57-69

O'Day-Bowman MB, Mavrogianis PA, Reuter LM, Johnson DE, Fazleabas AT and Verhage HG (1996) Association of oviduct specific glycoproteins with human and baboon (Papio anubis) ovarian oocytes and enhancement of human sperm binding to human hemizonae following in vitro incubation Biology of Reproduction 54 60-69

Oliphant G, Reynolds AB, Smith PF, Ross PR and Marta JS (1984) lmmunocytochemical localization and determination of hormone-induced synthesis of the sulfated oviductal glycoproteins Biology of Reproduction 31 165-174

Rapisarda JJ, Mavrogianis PA, O'Day-Bowman MB, Fazleabas AT and Verhage HG (1993) Immunological characterization and immunocytochemical localization of an oviduct-specific glycoprotein in the human Journal of Clinical Endocrinology and Metabolism 76 1483-1488

Reuter LM, O'Day-Bowman MB, Mavrogianis PA, Fazleabas AT and Verhage HG (1994) In vitro incubation of golden (Syrian) hamster ovarian oocytes and human sperm with a human oviductal specific glycoprotein Molectior Reproduction and Development 38 160-169

Robitaille G, St-Jacques S, Potier M and Bleau G (1988) Characterization of an oviductal glycoprotein associated with the ovulated hamster oocyte Biology of Reproduction 38 687-694

Schmidt A, Mavrogianis PA, O'Day-Bowman MB and Verhage HG (1997) Species-specific effect of oviductal glycoproteins on hamster sperm binding to hamster oocytes Molecular Reproduction and Development 46 201-207

Staros AL and Killian GJ (1997) Improvement of in vitro fertilization rates following treatment of oocytes with the bovine estrus-associated protein (EAP) Biology of Reproduction $\mathbf{5 6}$ Supplement $\mathbf{1} 86$

Thibault C (1973) Sperm transport and storage in vertebrates Joumal of Reproduction and Fertility Supplement 18 39-53

Thibodeaux JK, Myers MW, Prough SG and White KL. (1995) Effect of a serum extender containing growth factors on development of IVM and IVF bovine embryos Theriogenology 44 423-432

Towbin H, Stachelin T and Gordon J (1979) Electrophoretic transfer of proteins from polyacrylamide gels to nitrocellulose sheets: procedure and some applications Proceedings of National Academy of Sciences USA 76 4350-4354

Verhage HG, Fazleabas AT and Donnelly KM (1988) The in vitro synthesis and release of proteins by the human oviduct Endocrinology 1221639 1645

Verhage HG, Boice ML, Mavrogianis PA, Donnelly K and Fazleabas AT (1989) Immunological characterization and immunocytochemical localization of oviduct-specific glycoproteins in the baboon (Papio anubis). Endocrinology 124 2464-2472

Verhage HG, Fazleabas AT, Mavrogianis PA, O'Day-Bowman MB, Donnelly KM, Arias EB and Jaffe RC (1997) The baboon oviduct: characteristics of an estradiol-dependent oviduct-specific glycoprotein Human Reproduction Update 3 541-552

Wegner CC and Killian GJ (1991) In vifro and in vivo association of an oviduct estrus-associated protein with bovine zona pellucida Molecular Reproduction and Development 29 77-84 